\title{
Star Formation in Young Cluster NGC 1893
}

\author{
Saurabh Sharma ${ }^{1}$, A. K. Pandey ${ }^{2,1}$, D. K. Ojha ${ }^{3}$, W. P. Chen ${ }^{2}$, \\ S. K. Ghosh ${ }^{3}$, B. C. Bhatt ${ }^{4}$, G. Maheswar ${ }^{1}$ and Ram Sagar ${ }^{1}$ \\ ${ }^{1}$ Aryabhatta Research Institute of Observational Sciences (ARIES), Nainital, 263 129, India; \\ saurabh@aries.ernet.in \\ ${ }^{2}$ Institute of Astronomy, National Central University, Chung-Li 32054, Taiwan \\ ${ }^{3}$ Tata Institute of Fundamental Research, Mumbai (Bombay) - 400 005, India \\ ${ }^{4}$ CREST, Indian Institute of Astrophysics, Hosakote 562 114, India
}

\begin{abstract}
We have carried out a multi-wavelength study of the star forming region NGC 1893 to make a comprehensive exploration of the effects of massive stars on low mass star formation. Using deep optical $U B V R I$ broad band, $\mathrm{H} \alpha$ narrow band photometry and slit-less spectroscopy along with archival data from the surveys such as 2MASS, MSX, IRAS and NVSS, we have studied the region to understand the star formation scenario in the region.
\end{abstract}

Keywords. techniques: photometric, stars: formation, stars: luminosity function, mass function, stars: pre-main-sequence

\section{Introduction}

Young open clusters provide important information relating to star formation process and stellar evolution, because such clusters contain massive stars as well as low mass pre-main sequence (PMS) stars. Advancement in detectors along with various surveys such as the 2MASS, DENIS, ISO and Spitzer have permitted detailed studies of low-mass stellar population in regions of high mass star formation.

The very young open cluster NGC 1893 (Massey et al. 1995) is considered to be the center of the Aur OB2 association. NGC 1893 can be recognized as an extended region of loosely grouped early-type stars, associated with the H II region IC 410 with two pennant nebulae, Sim 129 and Sim 130 (Gaze \& Shajn 1952) and obscured by several conspicuous dust clouds. NGC 1893 contains at least five O-type stars, two of which, HD 242908 and LS V $+33^{\circ} 16$ are main-sequence O5 stars (Marco \& Negueruela 2002). The detection of PMS objects in NGC 1893 is interesting because it is one of the youngest known open cluster and has a moderately large population of O-type stars, representing thus a good laboratory for the study of massive star formation and the impact of massive stars on the formation of lower-mass stars (Marco \& Negueruela 2002).

\section{Results}

Deep optical $U B V R I$ and narrow band $H \alpha$ photometric data were taken using 1 meter Sampurnanand Telescope of ARIES, Nainital, India. The cluster region was also observed in the slitless mode with a grism as the dispersing element using the Himalayan Faint Object Spectrograph Camera (HFOSC) instrument mounted on 2-meter Himalayan Chandra Telescope at Hanle. Archival data from the surveys such as 2MASS, MSX, IRAS and NVSS are also used to understand the star formation scenario in and around the cluster region. 


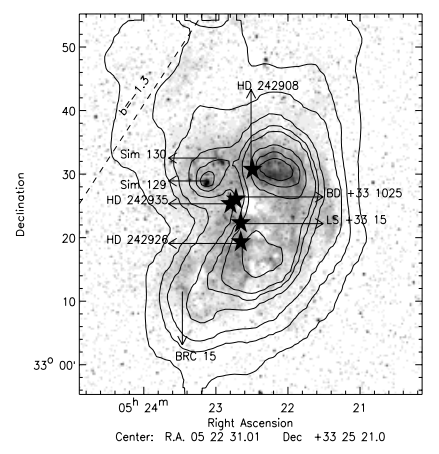

Figure 1. The $1^{\circ} \times 1^{\circ}$ R-band image of the field containing NGC 1893 , Sim 129 \& Sim 130 is reproduced from Digitized Sky Survey (DSS). North is up and east is to the left. Contours of $100-\mu \mathrm{m}$ flux from IRAS $100-\mu \mathrm{m}$ image is over-plotted. The IRAS $100-\mu \mathrm{m}$ image is smoothened. The contours are drawn at 60 (outer most), 80, 100, 150, 180, 200, 220, 250, 280 and $300 \mathrm{MJy} \mathrm{sr}^{-1}$. Locations of five O-type stars towards NGC 1893 are identified and shown using filled star symbols.

Reddening $(E(B-V))$ in the direction of cluster is found to be varying between 0.40 to $0.60 \mathrm{mag}$. The post-main-sequence age and distance of the cluster are found to be $\sim 4$ Myr and $3.25 \pm 0.20 \mathrm{kpc}$ respectively. Using the NIR two colour diagram and excess $H \alpha$ emission we identified candidate YSOs which are aligned from the cluster to the direction of nebula Sim 129 (see Fig. 1). The stars closer to Sim 129 and 130 show an average age of $\sim 1.5 \mathrm{Myr}$ which is smaller than the estimated age of the cluster $(\sim 4 \mathrm{Myr})$. The O-type stars at the center of the cluster may be responsible for the trigger of star formation in the region. The morphology of the cluster seems to be influenced by the star formation in the region. The PMS YSOs in the cluster region have masses $\sim 1-3.5 M_{\odot}$. The position of the YSOs on the CMDs indicates that the majority of these stars have ages between $\sim 1$ Myr to 5 Myr indicating a possibility of non-coeval star formation in the cluster.

The power law slope of the K-band luminosity function for the cluster is found to be $0.34 \pm 0.08$ which is consistent with the average value $(\sim 0.4)$ obtained for young star clusters (Lada et al. 1991; Lada \& Lada 1995; Lada \& Lada 2003). The slope of the initial mass function ' $\Gamma$ ' for PMS stars (mass range $0.6<M / M_{\odot} \leqslant 2.0$ ) is found to be $-0.88 \pm 0.09$, which is shallower than the value $(-1.71 \pm 0.20)$ obtained for MS stars having mass range $2.5<M / M_{\odot} \leqslant 17.7$. However for the entire mass range $\left(0.6<M / M_{\odot} \leqslant 17.7\right)$ the ' $\Gamma$ ' comes out to be $-1.27 \pm 0.08$. The effect of mass segregation can be seen on the MS stars. The estimated dynamical evolution time is found to be greater than the age of the cluster, therefore the observed mass segregation in the cluster may be the imprint of the star formation process.

\section{References}

Gaze, V. F. \& Shajn, G. A., 1952, Izv. Krym. Astrofiz. Obs., 9, 52

Lada, E. A., Evans, N. J. II, Depoy, D. L., \& Gatley, I., 1991, ApJ, 371, 171

Lada, E. A. \& Lada, C. J., 1995, AJ, 109, 1682

Lada, C. J. \& Lada, E. A., 2003, ARA $\& A, 41,57$

Marco, A. \& Negueruela, I., 2002, A\&A, 393, 195

Massey, P., Johnson, K. E., \& DeGioia-Eastwood, K., 1995, ApJ, 454, 151 